\title{
Research on the relationship between the rail corrugation and the vibration response of the wheel-rail system
}

\author{
Xinyu Jia ${ }^{1, a}$, Guoqing Li ${ }^{2}$, Xiubo Liu² and Peng Chen ${ }^{1}$ \\ ${ }^{1}$ China Academy of Railway Sciences, Beijing, China \\ 2 Infrastructure Inspection Research Institute, China Academy of Railway Sciences Corporation Limited, Beijing, China
}

\begin{abstract}
Rail corrugation is a very common phenomenon in high-speed railways. Rail corrugation can cause increased vibration of trains and tracks, and may even affect the safety of train operation. Therefore, this paper will analyze the vibration of the wheel-rail system caused by the rail corrugation. The actual transient rolling contact model is established by using explicit and implicit finite element methods. Through the calculation of the wheel-rail contact force and the wheel-rail mode, the vibration relationship between the rail corrugation and the wheel-rail system is obtained. It can provide some references for further analysis of the cause of rail corrugation.
\end{abstract}

\section{Introduction}

Rail corrugation is widely found in the railway sector. Whether it is high-speed railway, urban rail transit or heavy haul railway, there is the phenomenon of rail corrugation. Rail corrugation is a kind of short-wave periodic irregularity, which mainly occurs on the top surface of the rail. As shown in Fig. 1, it is rail corrugation that appears on a high-speed railway. Once rail corrugation occurs, the vibration of the vehicle and the track will be intensified, which will make the passenger feel uncomfortable and will also worsen the wheel and the rail, and even affect the safety of driving[1, 2 ]. Therefore, it is of great significance to study the intrinsic characteristics of corrugation and analyze the vibration law of wheel-rail system caused by corrugation, which will reduce the influence of corrugation and promote the healthy development of high-speed railway.

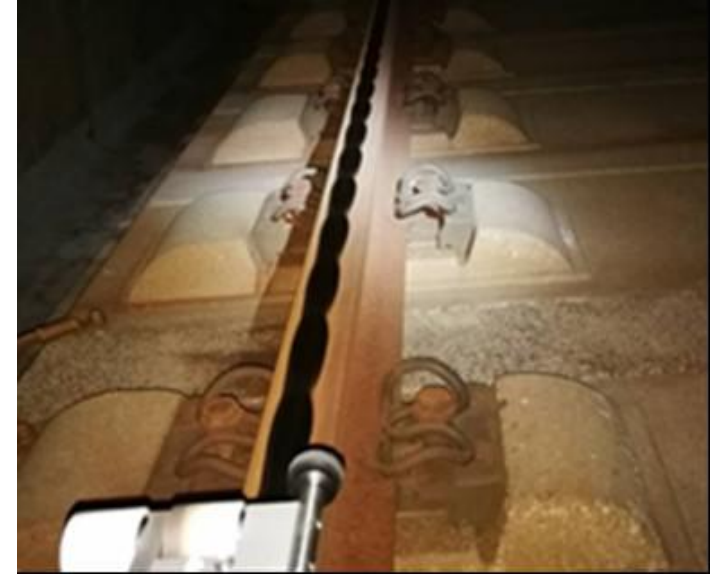

Fig. 1 High-speed railway rail grinding
Grassie[3] summarized the characteristics, causes and treatment methods of rail corrugation, and classified the rail corrugation in different states and discussed their characteristics separately. Knothe[4] proposed to have a deeper understanding of the formation mechanism of rail corrugation, and must have a deeper understanding of the transient wheel-rail rolling contact.

In the previous research on corrugation, it is often impossible to analyze the real wheel-rail contact model, which is a corresponding simplification of the wheel-rail system. For example, the contact between the wheel and the track is simulated using a Hertz spring, the track is simplified to a beam, and the wheel is simplified to a mass point $[5,6]$. These methods can provide a good reference for us to understand the corrugation, but many problems cannot be solved. The finite element method can simulate the relationship between the wheel and rail more realistically. Zhao[7, 8] and so on have verified the finite element simulation of wheel-rail contact.

In this paper, an explicit and implicit combination method is used to simulate three-dimensional transient rolling contact, analyze the vibration response of the wheel rolling over the rail corrugation section. By analyzing the relationship between wheel-rail vibration response and corrugation, it provides a certain reference for understanding the cause of rail corrugation.

\section{Finite element model}

In this paper, the three-dimensional transient rolling contact finite element model is established by ANSYS/LS-DYNA software. The model can analyze the relationship between high-speed train and ballastless

\footnotetext{
${ }^{a}$ Corresponding author: jiaxinyu_cars@163.com
} 
track. The model consists of spring mass, primary suspension, wheel, rails, fastenings, slab and mortar layers, as shown in Fig. 2. The wheel profile is selected as the $\mathrm{LM}_{\mathrm{A}}$ type, the profile of the rail top is CN60, and the rail inclination is 1:40. In China's high-speed railway, the minimum curve radius of the line is $7000 \mathrm{~m}$, the traverse of the vehicle can be controlled within a very small range, and the length of the model analyzed in this paper is also short, therefore, regardless of the lateral displacement of the wheel, only the forward movement along the straight line is analyzed. In order to reduce the number of elements and improve the efficiency of calculation, this paper only uses half of the wheels and tracks for analysis.
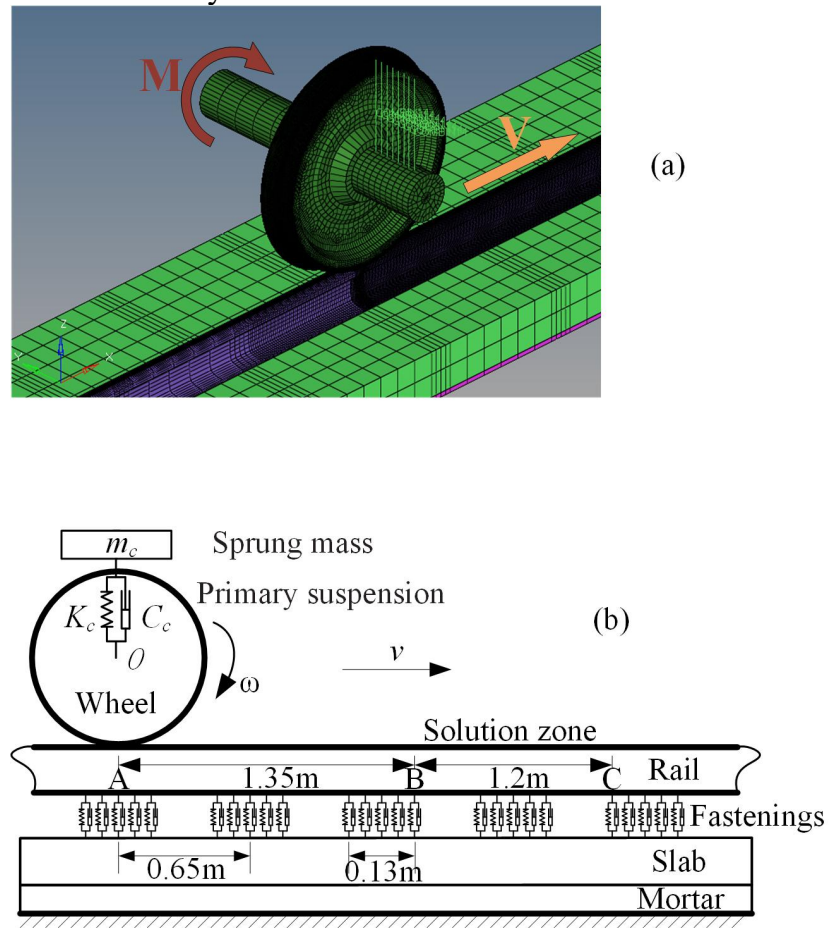

(a) Finite element mesh;(b) Dimension diagram

Fig. 2 Three-dimensional transient rolling contact finite element model

Wheel-rail vibration caused by rail corrugation grinding is a high-frequency vibration. The train above the primary suspension is less affected by high frequency vibration. Therefore the train above the primary suspension is simplified to a mass, and the value of the mass is determined by the axle weight of the train. The primary suspension consists of 8 parallel spring-damped structures for connecting the mass and the wheel axle. The contact between the wheel and the track uses a faceto-face contact criterion based on a penalty function. The friction between the contact faces is Coulomb's law with a coefficient of friction of 0.5 .

From point $\mathrm{A}$ to point $\mathrm{B}$ is a dynamic relaxation zone with a distance of $1.35 \mathrm{~m}$ to ensure that the wheel can roll smoothly. From point $\mathrm{B}$ to point $\mathrm{C}$ is a calculation zone, and a corrugation of $1.2 \mathrm{~m}$ in length is applied to the top surface of rail, in this section. The total length of the track is $13.5 \mathrm{~m}$, including 20 sleepers. In the dynamic relaxation zone and the calculation zone, the division of the rail elements is relatively fine, and the division away from the wheel rolling is relatively sparse. At the starting point of the wheel, point $A$, the minimum element size of the rail is $1 \mathrm{~mm}$; The minimum element size in the dynamic relaxation zone is $2.5 \mathrm{~mm}$; The minimum element size in the calculation zone is $1 \mathrm{~mm}$;

The fasteners were simulated with 5 × 5 spring damping structures ( 5 in the lateral direction and 5 in the longitudinal direction of the track). The arrangement of the springs is evenly distributed according to the size of the rail pad, and each rail pad has a size of $0.13 \mathrm{~m}$. The distance between the two sleepers is $0.65 \mathrm{~m}$. Both the slab and the mortar layer are simulated by elastic solid elements, and the material parameters are determined according to the measured values. Each slab is $6.5 \mathrm{~m}$ long and the distance between the slabs is $50 \mathrm{~mm}$, which is filled with concrete.

The $\mathrm{x}$-axis of the model is longitudinal, that is, the direction in which the wheel rolls; The y-axis of the model is horizontal and the y-axis is forward pointing to the inside of the track; The $\mathrm{z}$-axis of the model is vertical. The boundary conditions of the model are as follows: The mass point is coupled to the axle to ensure that the mass point has only a vertical movement relative to the wheel axle. A lateral symmetry constraint is applied to the end of the wheel axle. A symmetrical constraint is applied to both ends of the rail. The fasteners, slabs and mortar layers are restrained in the horizontal and vertical directions to ensure that they can only move in the vertical direction. The bottom of the mortar layer is fixed. $1[9]$.

The parameters used in the model are shown in Table

\begin{tabular}{lcc}
\multicolumn{2}{c}{ Table 1 Values of parameters } \\
\hline \multicolumn{2}{c}{ Parameter } & Value \\
\hline Sprung mass, $m_{\mathrm{c}}(\mathrm{kg})$ & 8000 \\
Stiffness of primary suspension, $K_{\mathrm{c}}(\mathrm{kN} / \mathrm{m})$ & 880 \\
Damping of primary suspension, $C_{\mathrm{c}}(\mathrm{kN} \cdot \mathrm{s} / \mathrm{m})$ & 4 \\
Stiffness of fastenings, $K_{\mathrm{f}}(\mathrm{MN} / \mathrm{m})$ & 22 \\
Damping of fastenings, $C_{\mathrm{f}}(\mathrm{kN} \cdot \mathrm{s} / \mathrm{m})$ & 200 \\
& Young's modulus, $E(\mathrm{GPa})$ & 205.9 \\
Material of & Poisson's ratio, $v$ & 0.3 \\
wheel \& rail & Density, $\rho\left(\mathrm{kg} / \mathrm{m}^{3}\right)$ & 7790 \\
& Young's modulus, $E(\mathrm{GPa})$ & 34.5 \\
Material of slab & Poisson's ratio, $v$ & 0.25 \\
& Density, $\rho\left(\mathrm{kg} / \mathrm{m}^{3}\right)$ & 2400 \\
& Young's modulus, $E(\mathrm{GPa})$ & 29.5 \\
Material filled & Poisson's ratio, $v$ & 0.25 \\
in slab gaps & Density, $\rho\left(\mathrm{kg} / \mathrm{m}^{3}\right)$ & 2400 \\
& Young's modulus, $E(\mathrm{GPa})$ & 8 \\
Mortar material & Poisson's ratio, $v$ & 0.2 \\
& Density, $\rho\left(\mathrm{kg} / \mathrm{m}^{3}\right)$ & 1600 \\
\hline
\end{tabular}

The calculation process of the model can be divided into three steps, (1) stress initialization, (2) dynamic relaxation, (3) computational solution. In the first step, the implicit solution method is used to calculate the 
deformation displacement of the wheel-rail system under the action of gravity when the wheel is placed on the track. In the second step, the displacement field obtained in the first step is applied to the corresponding node as the initial deformation. At the same time, the translational speed and the rotational speed are applied to the wheel at $\mathrm{t}=0$ as an initial condition. The transient rolling contact and the dynamic behavior of the wheel-rail system is calculated by the explicit solver solution method. From point A to point B in Fig. 2(b) is the dynamic relaxation zone. The reason for setting this region is that the calculation methods of implicit solution and explicit solution are different. The conversion of an implicit solution to an explicit solution introduces numerical instability that can result in unreal high-frequency vibrations in the wheel-rail system. When the wheel rolls over the dynamic relaxation zone, the wheel and rail will reach an approximately steady-state rolling, and the unreal high-frequency vibration will also disappear under the action of damping, thus ensuring that the obtained result is effective and reliable when entering the calculation solution zone. The third step is to calculate the various response results when the wheel rolls over the rail corrugation.

Among them, the wheel-rail contact force, the acceleration of the wheel and the track are common indicators. The schematic diagram of the calculation process is shown in Fig.3.

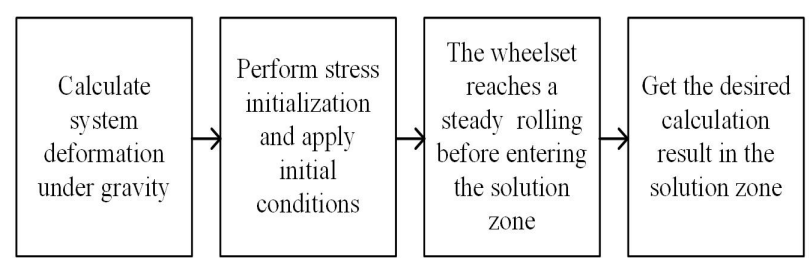

Fig. 3 A schematic diagram of the calculation process.

Under the action of gravity, the stress cloud diagram of the rail when the wheel pair stays on the rail is shown in Fig. 4. There is an elliptical contact spot at the rail top position of the rail, and the maximum stress value in the contact patch is $1.325 \times 10^{9} \mathrm{~Pa}$.

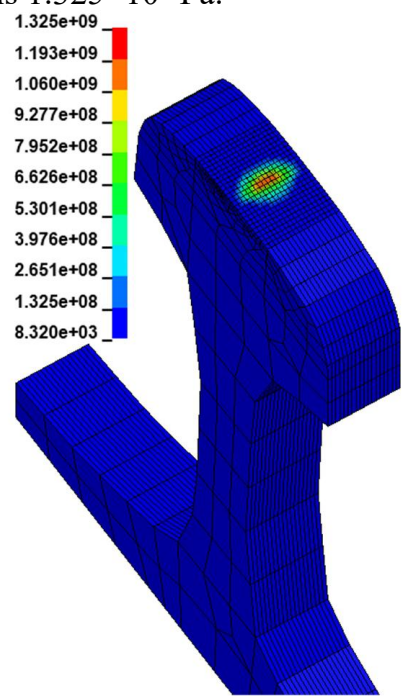

Fig. 4 The stress cloud diagram of rail

Subsequently, the wheel-rail contact force when the wheel rolled over the smooth rail was analyzed, as shown in Fig. 5. The curve in the figure shows the change of the vertical wheel-rail contact force with time, and the points $\mathrm{A}, \mathrm{B}$ and $\mathrm{C}$ are the points in Fig. 2. It can be seen from the figure that there is a high-frequency vibration of the wheel-rail contact force from the point $\mathrm{A}$ to the point $\mathrm{B}$, which is due to the inevitable vibration of the wheelset from static to dynamic. As time increases, the amplitude of the vibration of the wheelset is gradually reduced by the damping. When point $\mathrm{B}$ is reached, the wheelset is basically in a steady rolling state. From point $\mathrm{B}$ to point $\mathrm{C}$, the wheel-rail force is basically stable and coincides with the axle weight, which can be used for further analysis and calculation.

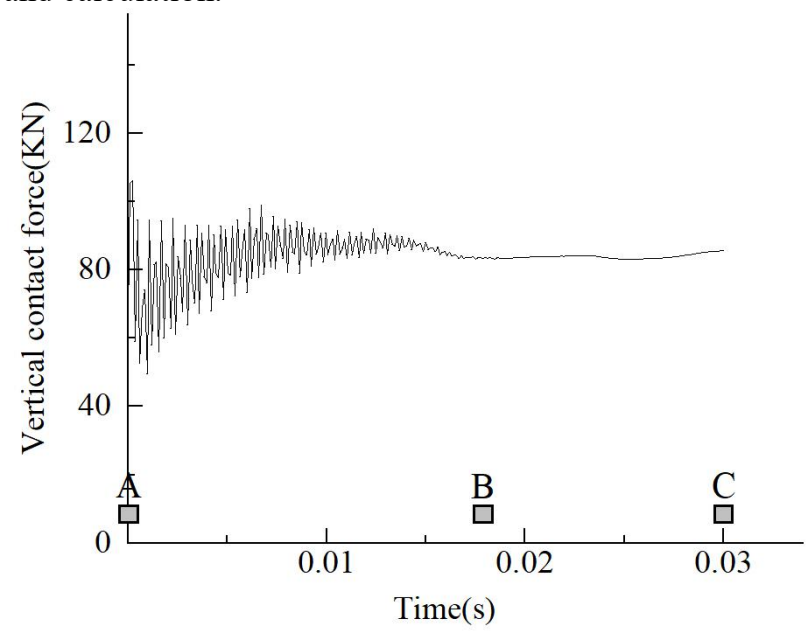

Fig. 5 Vertical wheel-rail contact force

\section{Vibration response caused by rail corrugation}

First, select a high-speed line containing the rail corrugation, measure the rail corrugation, and obtain the data such as the wavelength and depth, as shown in Fig. 6, the length of the rail corrugation is $1 \mathrm{~m}$.

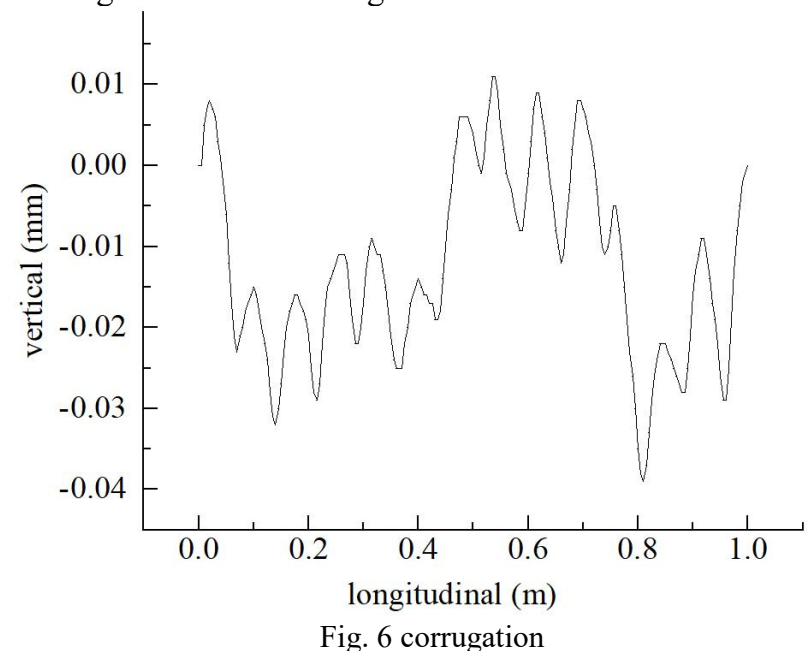

The wave curve is Fourier transformed to obtain a spectrogram, and the frequency characteristics of the curve are analysed, as shown in Fig. 7. 


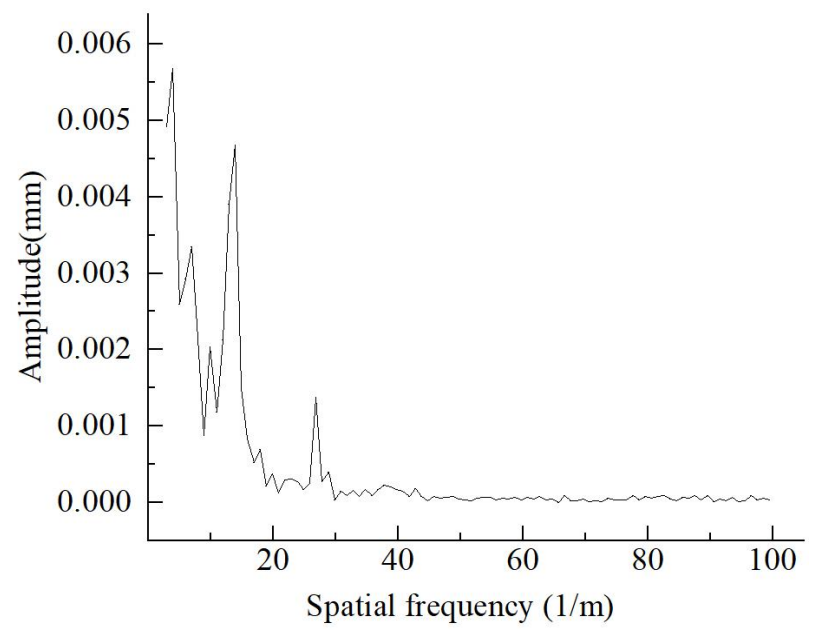

Fig. 7 Spatial frequency of the rail corrugation

The curve in Fig. 6 is applied to the top surface of the rail in the solution zone to obtain the wheel-rail contact force, as shown in Fig. 8. It can be seen from the figure that the wheelset enters the rail corrugation section at $0.017 \mathrm{~s}$ and exits the rail corrugation section at $0.03 \mathrm{~s}$.

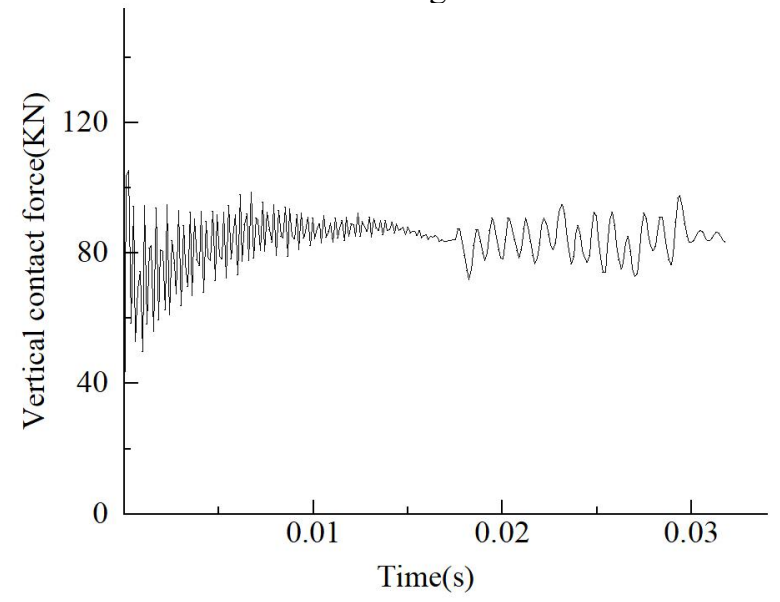

Fig. 8 Wheel-rail contact force

Fourier changes were made to the wheel-rail contact force of the rail corrugation section to analyze the frequency characteristics, as shown in Fig. 9. It can be seen from the figure that the frequency is mainly concentrated in $0 \sim 1220 \mathrm{HZ}$ and near $2220 \mathrm{HZ}$, and the maximum amplitude occurs at the frequency of $1155 \mathrm{HZ}$.

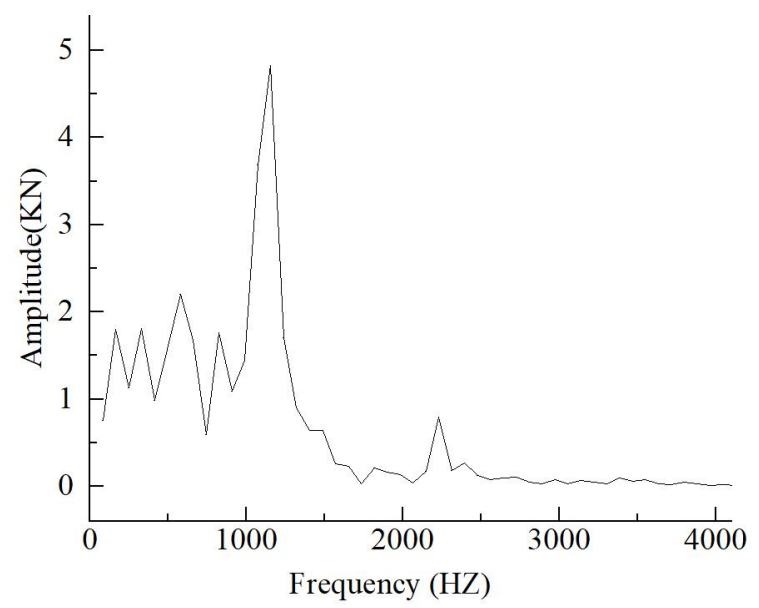

Fig. 9 Spectrogram of wheel-rail contact force.
In order to obtain the frequency characteristic relationship between the wheel-rail force and the rail corrugation, the two frequencies are put together for comparison, and the spatial frequency of the rail corrugation is multiplied by the speed of the wheelset to obtain the excitation frequency to the wheel, as shown in Fig. 10.

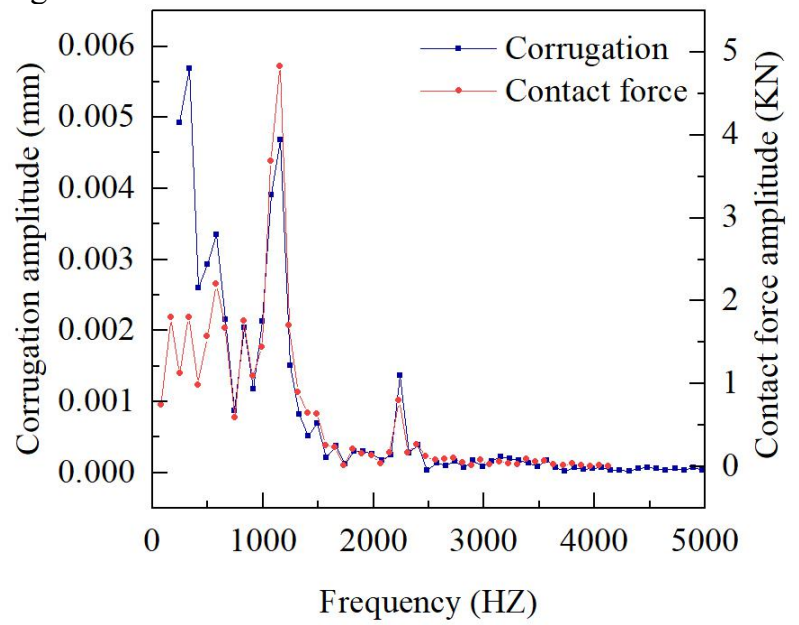

Fig. 10 Spectrogram of rail corrugation and wheel-rail contact forces.

Modal analysis is performed on the wheel and the track respectively. The track contains rails, fasteners, slabs and mortar layer, and the bottom of the mortar layer is fixed. In order to save space, only the first few modal values of the wheel and the track, and the modal values in the frequency range with larger amplitude in Fig. 10 are listed. The modes of the wheel and track are shown in Table 2.

Table 2 Vibration mode of wheel and track

\begin{tabular}{cccc}
\hline \multicolumn{2}{c}{ Wheelset } & \multicolumn{2}{c}{ Rail } \\
\hline Mode number & Frequency & Mode number & Frequency \\
3 & 0 & 1 & 51.26 \\
5 & 0 & 2 & 65.18 \\
6 & 0 & 3 & 82.07 \\
7 & 0 & 4 & 101.77 \\
8 & 300 & 20 & 285.92 \\
9 & 300 & 21 & 304.86 \\
10 & 448 & 22 & 335.86 \\
11 & 448 & 23 & 340.71 \\
12 & 473.51 & 33 & 574.69 \\
13 & 731.57 & 34 & 595.29 \\
14 & 731.57 & 35 & 611.83 \\
15 & 1114.1 & 49 & 1041.2 \\
16 & 1114.1 & 50 & 1098.6 \\
17 & 1146.2 & 51 & 1157.8 \\
\hline 16 & 1439.5 & 52 & 1207.7 \\
\hline
\end{tabular}

It can be seen from the Table 2 that in the vicinity of the frequency at which the amplitude of the wave is largest $(333 \mathrm{HZ})$, only the $22 \mathrm{nd}$ mode of the track 
(335.86HZ) is relatively close, and the wheel has no close value. At a frequency of $581 \mathrm{~Hz}$, the amplitude of the rail corrugation has reached a peak, but in this frequency range, only the $33 \mathrm{rd}$ mode of the track $(574.69 \mathrm{HZ})$ is relatively close, and the wheel has no close frequency. Near the frequency $(1155 \mathrm{HZ})$ where the wheel-rail contact force amplitude is the largest, the 16th mode of the wheel $(1146.2 \mathrm{HZ})$ and the 51st mode of the track $(1157.8 \mathrm{HZ})$ are close to this value. It can be concluded that the vibration response of the wheel-rail system is determined by the external excitation and the mode of the wheel-rail system itself. When the excitation frequency of the rail corrugation is close to the natural frequency of the wheel-rail system, the frequency amplitude of the wheelrail contact force is maximized.

\section{Conclusions}

In order to analyze the influence of rail corrugation on the vibration of the wheel-rail system in high-speed railway, this paper adopts the explicit and implicit finite element method to establish the transient rolling contact model of the wheel rolling over the rail corrugation section, and calculate the wheel-rail contact force and vibration mode, and reached the following conclusions:

(1) The frequency of the measured wave grinding is in good agreement with the calculated frequency of the wheel-rail force. By analyzing the frequency of the wheel-rail contact force, the main frequency range of the rail corrugation can be obtained, which provides a certain reference for the detection of the rail corrugation.
(2) The vibration response of the wheel-rail system is determined by the excitation frequency of the rail corrugation and the natural frequency of the wheel-rail system itself. When the excitation frequency of the rail corrugation is close to the natural frequency of the wheelrail system, the frequency amplitude of the wheel-rail contact force is maximized.

\section{References}

1. S. Li, Z. Li, A. Núñez and R. Dollevoet, Applied Sciences, 7 (2017)

2. L. Zhou and Z.-y. Shen, Journal of Zhejiang University SCIENCE A, 14 (2013)

3. S. Grassie and J. Kalousek, Proceedings of the Institution of Mechanical Engineers, Part F: Journal of Rail and Rapid Transit, 207 (1993)

4. K. Knothe and A. Groß-Thebing, Vehicle system dynamics, 46 (2008)

5. J. C. Nielsen, Wear, 265 (2008)

6. W. Zhai, H. Xia, C. Cai, M. Gao, X. Li, X. Guo, N. Zhang and $\mathrm{K}$. Wang, International Journal of Rail Transportation, 1 (2013)

7. K. D. Vo, H. T. Zhu, A. K. Tieu and P. B. Kosasih, Wear, 322-323 (2015)

8. X. Zhao, J. Yang, B. An, C. Liu, Y. Cao, Z. Wen and $\mathrm{X}$. Jin, Proceedings of the Institution of Mechanical Engineers, Part F: Journal of Rail and Rapid Transit, 232 (2016)

9. X. Zhao, Z.-f. Wen, H.-y. Wang, X.-s. Jin and M.-h. Zhu, Journal of Zhejiang University SCIENCE A, 15 (2014) 